\title{
1 Rapid, but limited, zooplankton adaptation to simultaneous warming and 2 acidification
}

3 Hans G. Dam ${ }^{1,5^{*}}$, James A. deMayo ${ }^{1,5,}{ }^{*}$, Gihong Park ${ }^{1}$, Lydia Norton ${ }^{1}$, Xuejia $\mathrm{He}^{2}$, Michael B.

$4 \quad$ Finiguerra $^{3}$, Hannes Baumann ${ }^{1}$, Reid S. Brennan ${ }^{4}$, Melissa H. Pespeni ${ }^{4}$.

5

6 1. Department of Marine Sciences, University of Connecticut, Groton, CT 06340-6048, USA

7

8 2. Research Center for Harmful Algae and Marine Biology, Jinan University,

9 Guangzhou 510632, Guangdong Province, P.R. CHINA

11 3. Department of Ecology and Evolutionary Biology, University of Connecticut, Groton, CT

12 06340-6048, USA

13

14 4. Department of Biology, the University of Vermont, Burlington, VT 05405, USA

15

16 5. Equal contribution

17

*Communicating authors

20 Author contact information:

21 Hans G. Dam: hans.dam@uconn.edu

22 James A. deMayo: james.demayo@uconn.edu

23 Gihong Park: gihong.park@uconn.edu

24 Lydia Norton: 1ydia.norton@uconn.edu

25 Xuejia He: hexuejia@sina.com

26 Michael B. Finiguerra: michael.finiguerra@uconn.edu

27 Hannes Baumann: hannes.baumann@uconn.edu

28 Reid S. Brennan: reid.brennan@uvm.edu

29 Melissa H. Pespeni: melissa.pespeni@uvm.edu 
30 Abstract: Predicting the response of marine metazoans to climate change is hampered by a lack

31 of studies on evolutionary adaptation, particularly to combined ocean warming and acidification

32 (OWA). We provide evidence for rapid adaptation to OWA in the foundational copepod species,

33 Acartia tonsa, by assessing changes in population fitness based on a comprehensive suite of life-

34 history traits, using an orthogonal experimental design of nominal temperature $\left(18^{\circ} \mathrm{C}, 22^{\circ} \mathrm{C}\right)$ and

$35 p \mathrm{CO}_{2}(400,2000 \mu \mathrm{atm})$ for 25 generations ( 1 year). Egg production and hatching success

36 initially decreased under OWA, resulting in a 56\% reduction in fitness. However, both traits

37 recovered by the third generation and average fitness was reduced thereafter by only $9 \%$.

38 Antagonistic interactions between warming and acidification in later generations decreased

39 survival, thereby limiting full fitness recovery. Our results suggest such interactions constrain

40 evolutionary rescue and add complexity to predictions of the responses of metazoan populations

41 to climate change. 
44 The continuous increase in atmospheric $\mathrm{CO}_{2}$ is unparalleled in the last 300 million years ${ }^{1}$,

45 causing rapid ocean warming $(\mathrm{OW})^{2,3}$ and ocean acidification $(\mathrm{OA})^{4}$. Warming and acidification

46 in coastal regions are projected to be more extreme $e^{5,6}$. Overall, novel environments may arise as

47 a result of these changes ${ }^{7}$. Predicting how biota respond to this rapid global change is a crucial

48 yet formidable scientific challenge, particularly whether populations can persist at similar levels

49 and phenotypes in the future ${ }^{8}$. Organisms can respond to novel environments through phenotypic

50 plasticity or evolutionary adaptation, both of which can mitigate the deleterious effects of climate

51 change ${ }^{8-12}$. In particular, long-term experimental evolution studies are a powerful tool to

52 examine the role of adaptation in mitigating the effects climate change on biota and to explore

53 whether populations can evolve fast enough to keep pace ${ }^{13}$.

55 Organismal performance typically decreases sharply when temperatures increase beyond the

56 optimum ${ }^{14}$ leading to disproportional deleterious effects on performance. In addition, marine

57 metazoans require the mobilization of energy-demanding acid-base regulatory processes to

58 counteract internal $\mathrm{pH}$ reduction to maintain homeostasis under high $\mathrm{CO}_{2}$ conditions. This may

59 result in increased metabolic costs at the expense of growth and reproduction, even for non-

60 calcifying metazoans ${ }^{15-17}$. Although factorial assessments of species sensitivities to warming and

61 acidification have increased rapidly over the past years, multi-generational studies on metazoan

62 populations responding to future simultaneous warming and acidification (OWA) are rare ${ }^{18-20}$.

63 Moreover, lack of population fitness measures in existing studies preclude considerations of

64 evolutionary rescue - evolution occurring sufficiently fast to allow population recovery before

65 extirpation $^{21-23}$. 
We used an experimental evolution approach to test whether a marine zooplankter, the copepod

Acartia tonsa (Dana; 1849), can adapt to environments created by OW, OA, and OWA conditions, to identify the functional traits under selection, and to assess evolutionary rescue. As the most abundant metazoans on the planet ${ }^{24,25}$, copepods link primary producers and other microbes to upper trophic levels, thereby influencing fisheries productivity ${ }^{26,27}$ and mediating marine biogeochemical cycles ${ }^{28}$. Specifically, Acartia tonsa is a dominant copepod in estuarine

72 systems from tropical to temperate regions ${ }^{29}$ and a main prey item of forage fish ${ }^{30}$, which makes

73 this species an important zooplankton model. Using both improvements in trait performance and

74 population fitness across generations, we show rapid, yet limited copepod adaptation to

75 combined ocean warming and acidification likely driven by an antagonistic interaction of 76 warming and acidification.

79 We measured five fitness-relevant life-history traits (survival, egg production rate (EPR), egg hatching success (HS), development time, and sex ratio) across 25 generations in an orthogonal

81 design with two levels of $\mathrm{CO}_{2}$ and temperature. A population of Acartia tonsa was collected

82 from Long Island Sound $\left(41.3^{\circ} \mathrm{N}, 72.0^{\circ} \mathrm{W}\right.$; Groton, CT, USA) and kept under standard

83 laboratory conditions (see methods) for at least three generations prior to the experiment. Four

84 lines of the population were established with four replicates of each condition. The target (actual

$85 \pm$ standard deviation) conditions were: ambient temperature $=18^{\circ} \mathrm{C}(18 \pm 0.34, \mathrm{~N}=330)$,

86 ambient $p \mathrm{CO}_{2}=400 \mu \operatorname{atm}(379 \pm 36, \mathrm{~N}=18 ; \mathrm{pH}=8.26 \pm 0.1, \mathrm{~N}=330)$; high temperature $=$

$8722^{\circ} \mathrm{C}(22 \pm 0.81, \mathrm{~N}=336)$, and high $p \mathrm{CO}_{2}=2000 \mu \operatorname{atm}(2301 \pm 215, \mathrm{~N}=18 ; \mathrm{pH}=7.55 \pm 0.08$,

$88 \mathrm{~N}=330$ ). Ambient target levels represented extant conditions for this species in NE Atlantic 
estuaries (see methods for choice of temperature), and high levels corresponded to future conditions based on global projections for the years $2100-2300^{1-4}$, although A. tonsa already periodically experiences high temperature and $\mathrm{CO}_{2}$ levels in its growth season in NE Atlantic estuaries $^{31,32}$. Summaries of the temperature, $\mathrm{pH}$, and $\mathrm{CO}_{2}$ data are shown in Supplementary Tables 1-3. Details of statistical tests and their significance for the transgenerational experiment are in the methods section.

\section{Evidence for adaptation}

During the first experimental generation (generation zero), EPR and HS declined in all three future (OW, OA, and OWA) conditions relative to ambient (AM) conditions: OW (EPR: $p<$ 0.0001; HS: $p<0.01$; t-test), OA (EPR: $p<0.0001$; HS: $p=0.19$; t-test), and OWA (EPR: < 0.0001; HS: $p<0.0001$; t-test; Fig. 1), illustrating the ecological effects of these climate change variables. The decrease was strongest under OWA, particularly for HS, indicating synergistic deleterious effects of temperature and $\mathrm{CO}_{2}$.

Significant interactions of temperature and $\mathrm{CO}_{2}$ on $\mathrm{HS}$ were evident across all generations $(p<$ 0.0001; three-way ANOVA). After the first generation, EPR decreased under AM $(p<0.0001$;

GAM ANOVA) and OA conditions ( $p<0.0001$; GAM ANOVA), but partially recovered in later generations. Meanwhile, HS remained stable (AM GAM ANOVA: $p=0.14$; OA GAM ANOVA: $p=0.064$; Fig. 1). Under OW, EPR decreased with generation $(p<0.0001$; GAM ANOVA) but did not recover. Meanwhile, HS increased for OW ( $p<0.0001$; GAM ANOVA; Fig. 1C) suggesting some degree of adaptation. By contrast, under OWA, EPR increased by 50\% 
111 ( $p<0.02$; GAM ANOVA) and HS doubled ( $p<0.0001$; GAM ANOVA; Fig. 1D) by generation

1123 , and the improvements were maintained until generation 25 . These changes yielded significant

113 effects on population fitness (Table 1) and are consistent with rapid adaptation.

115 Survival from freshly hatched nauplii to reproductively mature adults was independent of 116 treatment $(p>0.05$, two-way ANOVA)for the first 12 generations ( Fig. 2). By generation 15,

117 however, survival decreased by $30 \%$ under OW ( $p=0.02$, t-test $)$ and OWA $(p=0.01$, t-test $)$

118 relative to ambient conditions. Acidification alone elicited no decrease in survival $(p>0.1$, one-

119 way ANOVA). Although survival recovered in the OW treatment by generation 25, survival

120 decreased under OWA by an additional 50\% relative to generation 15 for the same treatment $(p<$

1210.001 , t-test), demonstrating an antagonistic interaction between $\mathrm{OW}$ and $\mathrm{OA}$ in later

122 generations ${ }^{33}$. Traits under selection are expected to increase towards phenotypic optima ${ }^{34-36}$,

123 thus survival does not appear to be a trait under selection for adaptation to OW, OA or OWA

124 conditions. High temperature resulted in faster development in both the OW and the OWA

125 treatments (Supplementary Fig. 1) with 22-24\% shorter development times than at ambient

126 temperature ( $p<0.0001$; two-way ANOVA). High $\mathrm{CO}_{2}$, in contrast, resulted in 5-6\% slower

127 development times than ambient $\mathrm{CO}_{2}$ across all generations ( $p<0.0001$; two-way ANOVA).

128 Thus, warming and acidification acted antagonistically on development time. Finally, a 1:1 sex

129 ratio remained unchanged for three of the four treatments (Supplementary Fig. 2). Under OWA,

130 however, the proportion of females decreased across generations, with a significantly lower

131 proportion in generation 25 than generation $0(p<0.01$; Tukey HSD). 
133 To understand adaptation not just in terms of individual traits, but with respect to population

134 fitness, we integrated all measured traits (survival, EPR, HS, development time, and sex ratio) to

135 estimate the net reproductive rate, $\operatorname{lambda}^{37}(\lambda)$, which is the fraction of the population replaced

136 in a generation (Fig. 3). In generation 0, OWA conditions resulted in a $56 \%$ reduction in $\lambda$

137 relative to ambient conditions, while OW and OA resulted in $23 \%$ and $13 \%$ reductions relative to

138 ambient conditions, respectively ( $p<0.01$, t-test; Fig. 3). However, by generation $3, \lambda$ in OWA

139 conditions had improved by $120 \%$ relative to generation $0(p<0.0001$; Tukey HSD; Fig. 3$)$ and

140 recovered to levels equal to ambient conditions ( $p=1.0$; Tukey HSD; Fig. 3), mainly driven by

141 improved hatching success (Fig. 1). The $\lambda$-frequency distribution shows an inflation of zero

142 values (Supplementary Fig. 3) due to the high abundance of mate pairs with low HS at

143 generation 0, particularly under OWA conditions. In later generations, as HS increased so did the

144 probability of non-zero $\lambda$, and this was especially evident under OWA conditions

145 (Supplementary Fig. 4). Thus, as generations progressed, selection under OWA conditions culled

146 off low-fitness individuals in the population. Lambda remained high in OWA conditions until

147 generation 15, after which there was a $19 \%$ reduction $(p<0.05$, t-test; Fig. 3$)$. This decrease was

148 driven by reduced survival (Fig. 2), which suggests an inability of Acartia tonsa to maintain

149 multiple optimal phenotypes, i.e., high hatching success and high survivorship, under OWA

150 conditions. While $\lambda$ was $22 \%$ lower under OWA than ambient conditions by generation 25 , it

151 was significantly higher than in generation 0 ( $p<0.0001$, t-test; Fig. 3), still consistent with

152 adaptation over time. In accord, significant effects of generation on $\lambda$ were evident under OW ( $p$

$153<0.04$; ANOVA) and OWA $(p<0.001$; ANOVA) conditions, but not under ambient $(p=0.681$;

154 ANOVA) or OA ( $p=0.212$; ANOVA) conditions (Table 1). The latter suggests that OA alone

155 was not a strong selective force in our study. 
157 Adaptation is evident when performance increases towards optimal phenotypes that increase

158 fitness over time ${ }^{34-36}$. Here, we observed improved performance and fitness under OW and

159 OWA conditions, but with important differences between the treatments: fitness was fully

160 recovered under OW, but not under OWA conditions. Acartia tonsa exhibits strong tolerance to

161 high temperatures, consistent with its seasonal dominance in the summer and wide-ranging

162 latitudinal distribution ${ }^{29,38}$. However, the combination of elevated temperature and $\mathrm{CO}_{2}$ are

163 known to affect ectotherm resource partitioning and energy distribution ${ }^{15}$, which might account

164 for the limited recovery under OWA conditions. During adaptation, multiple phenotypes are

165 expected to reach optimal levels of performance concurrently to yield the maximum possible

166 population fitness for a particular environment. We hypothesize that under OWA conditions

167 copepods could not sustain multiple optimized phenotypes, as evidenced by the observed

168 reduction in survival following HS and EPR recovery to levels equal to or greater than those of

169 ambient conditions. Under OW, copepods improved both EPR and HS (Fig. 1) and maintained

170 high survival (Fig. 2) across generations to yield the highest $\lambda$ level relative to all conditions by

171 generation 25 (Fig. 3). By contrast, under OWA conditions the improvements in EPR and HS

172 across generations yielded the highest $\lambda$ values between generations 3 and 12 but decreases in

173 survival reduced $\lambda$ afterwards. These results are consistent with adaptation via selection, while

174 simultaneously allowing maladaptive traits to persist ${ }^{35}$ under OWA conditions.

176 Genetic drift affects global, genome-wide patterns of genetic diversity, while selective processes

177 affect specific regions of the genome ${ }^{39,40}$. We tested for signatures of genetic drift caused by

178 potential bottlenecks by exploring patterns of nucleotide diversity $(\pi)$ among treatments using 
179 single nucleotide polymorphisms identified with pooled sequencing of genomic DNA from each

180 replicate of each treatment at generation 25 . We found that global levels of nucleotide diversity

181 were equivalent across treatment groups (Supplementary Figure 5; Wilcoxon Rank Sum test, $p$ >

182 0.05; average $\pi$ across treatments: AM: $0.0135 \pm 0.006$; OA: $0.0130 \pm 0.006$; OW: $0.0134 \pm$

183 0.006; OWA: $0.0138 \pm 0.006$ ), revealing no evidence of genetic bottlenecks or drift after 25

184 generations. In separate work, we observed divergence in allele frequencies and gene expression

185 at generation 20 between the AM and OWA lines ${ }^{41}$. Among the four lineages at generation 25,

186 allele frequency estimates indicate differentiation between the four lines despite the similar

187 levels of nucleotide diversity (our own unpublished observations). Altogether, the improvement

188 in traits and population fitness across generations coupled with the genetic differentiation

189 between the OWA and the AM treatments are consistent with evolutionary adaptation in the

190 OWA treatment.

\section{Traits affecting fitness}

To assess the contribution of each life-history trait to adaptation, we quantified the strength of

194 selection via the standardized linear selection coefficient of the fitness landscape ${ }^{42}$. High linear

195 coefficients of selection for a given fitness landscape suggest that a particular trait is under

196 selection, though not necessarily determining fitness. The coefficients are calculated from

197 multiple regression models of relative $\lambda$ against all changing life-history traits (see methods). We

198 found that HS, but not EPR or survival, was under selection and had a positive impact on fitness.

199 Specifically, relative $\lambda$ increased as a function of HS with standardized linear selection

200 coefficients $(\beta)$ of $0.91(\mathrm{AM}), 0.94(\mathrm{OA}), 0.96(\mathrm{OW})$, and $0.78(\mathrm{OWA})$ at generation $0(p<$

2010.0001 , ANOVA; Fig. 4A). In addition, $\beta_{\mathrm{HS}}$ was significantly different for all treatments between 
202

203

204

205

206

207

208

209

210

211

212

213

214 We also employed path analysis on structural equation models (SEMs) to identify the

215 hierarchical interactions of all life-history traits and the traits' effects on fitness ${ }^{43,44}$. High

216

217

218

219

220

221

222

generations 0 and 25 ( $p<0.0001$, ANOVA), indicating changing degrees of selection on HS for all treatments-between the first and last generations. By contrast, neither EPR or survival were significant at generation 0 (Fig. 4B, C; $p>0.1$, ANOVA) suggesting that selection acted in favor of higher HS, but not towards higher survival or EPR for the OW and OWA treatments. By generation 25, the impact of HS on relative fitness decreased relative to generation 0 by $88 \%$ and $10 \%$ for the OW and OWA treatments, respectively. Such decreases corresponded to a shift in phenotype distribution towards higher HS and suggested that selection had relaxed by generation 25 as HS within the population reached the phenotypic optimum (Fig. 4A). Finally, despite the significant decrease in sex ratio over the 25 generations in the OWA treatment (Supplementary

Fig. 2), sex ratio had no effect on relative fitness between generations 0 and 25 ( $p=1.0$, ANOVA), suggesting this trait was not under selection. correlation coefficients of SEMs indicate causality of a given trait's effect towards fitness. The path analysis revealed that HS had the largest effect on fitness of all life-history traits across treatments, with the exception of the OW treatment at generation 25, and was significant at both generations 0 and 25 (Table 2). Taken together, we conclude that selection on HS both under OW and OWA conditions was the critical factor in adaptation to those environments.

\section{Implications}


223 Our study highlights the need for global change studies to consider population fitness, in addition

224 to individual traits, as an integrative tool for measuring adaptation ${ }^{27,45}$. For example, in this

225 experiment using survival alone would have led to the erroneous conclusion of no adaptation

226 under any treatment. Similarly, estimating fitness based on EPR alone would have also led to the

227 erroneous conclusion that fitness decreased between generations 3 and 15 for AM. Despite this

228 decrease in EPR, fitness remained unchanged across generations for AM (Fig. 3). Likewise,

229 using EPR and HS alone would have missed the fitness decrease after generation 12 under OWA

230 conditions. At the same time, the tradeoffs of EPR and HS versus survival after generation 12

231 (Figs. 1 and 2) partly explain the limited evolutionary rescue under OWA conditions (Fig. 3).

The shifting interactive effects of combined ocean warming and ocean acidification through time highlight the need to evaluate long-term evolutionary studies with multiple stressors and underline the complexity that accompanies predicting organism responses to climate change ${ }^{33,46}$.

236 Previous work has shown that Acartia tonsa can adapt to lower $\mathrm{CO}_{2}$ levels $(800 \mu \mathrm{atm})$ than those

237 in our study ${ }^{46}$. Thus, it is conceivable that full evolutionary rescue to OWA can be achieved at

238 lower $\mathrm{CO}_{2}$ levels. Furthermore, previous research has shown that exposure to OA over multiple 239 generations improves EPR and selects for genes involved in RNA processing and regulation of 240 metabolism in copepods ${ }^{11,47}$. Forthcoming genomic work from our group will determine if 241 similar genes are also under selection for OW and OWA. Neither deleterious effects of OA nor 242 adaptation to OA alone were observed across generations in our study (Fig. 3). Thus, our results 243 highlight the evolutionary rescue and accompanying limitations that arise from multi-stressor 244 adaptation. Assuming additive effects of warming and acidification in the present study should 245 have led to full evolutionary rescue under OWA conditions, which was not observed (Fig. 3). 
246

247

248

249

250

251

252

253

254

255

256

257

258

259

260

261

262

263

264

265

266

267

268

Instead, OW and OA had synergistic deleterious effects on fitness on generation 0 , but antagonistic effects on generation 25 that counterbalance the individual effects of OW and OA (Supplementary Table 4). We suggest that the limited evolutionary rescue under OWA conditions in our study must have arisen from an antagonistic interaction between warming and acidification, as has been hypothesized earlier in polychaetes ${ }^{19,20,48}$ or for other co-occurring environmental stressors in bivalves ${ }^{49}$. Thus, since OW and OA are occurring simultaneously, even crude predictions of population performance under climate change should consider nonadditive effects of temperature and $\mathrm{CO}_{2}$ interactions on population fitness.

Our study, showing both improved trait performance and population fitness across generations, constitutes the first demonstration of rapid adaptation to global change conditions for a metazoan. We present evidence for adaptation to both warming and combined warming and acidification, but not acidification alone, based on standing genetic variation. Previous work has been limited to phytoplankton ${ }^{39}$ or documenting marine metazoan traits for limited generations without population fitness estimates ${ }^{18-20}$. Failing to account for adaptation potential may overestimate future population vulnerability ${ }^{45,50}$. For example, using the observed deleterious effects at generation 0 to predict future vulnerability would have estimated a fitness reduction for the OWA treatment relative to ambient treatment of $56 \%$ (Fig. 3). However, accounting for evolutionary rescue (generations 3-25) resulted in an average fitness reduction of only $9 \%$. At the same time, our results suggest that full evolutionary rescue under OWA conditions was not achieved (Fig. 3). This result is consistent with the hypothesis that adaptation to stress comes at a cost and that full recovery of populations to future climate conditions based on extant genetic variation is limited ${ }^{51}$, even though $A$. tonsa periodically experiences the warming and 
269 acidification conditions examined here ${ }^{31,32}$. The limited evolutionary rescue we observed also has

270 consequences for future oceanic systems under OWA conditions. Because adaptation to OWA

271 conditions appears to be costly even under the food replete conditions of our study, it is unclear

272 to what extent evolutionary rescue would occur under scenarios that predict lower resource

273 availability for zooplankton under climate change ${ }^{50}$. Limited evolutionary rescue, in turn, would

274 reduce prey availability for fish, thereby negatively affecting fish production ${ }^{50}$. Finally, since

275 copepods are a major vector of carbon transfer from the surface waters to the sea floor ${ }^{28}$, limited

276 evolutionary rescue would also reduce the efficiency of the biological carbon pump, with a

277 concomitant lower drawdown of $\mathrm{CO}_{2}$ from near surface waters.

\section{Conclusions}

280 We show evidence for metazoan evolutionary adaptation to combined warming and acidification

281 by explicitly assessing changes in population fitness based on a comprehensive suite of life-

282 history traits. Adaptation was evident in the fitness improvements for animals after a few

283 generations, and confirmed by independent evidence of genetic differentiation. The strength of

284 selection coefficients and the path analysis suggested the key trait under selection affecting

285 fitness in our study was hatching success. Evolutionary rescue was limited by the interaction of

286 warming and acidification, which switched from synergistic to antagonistic from the beginning

287 to the end of the experiment, adding complexity to predictions of population adaptation to

288 climate change. 
Methods

\section{a) Copepod culturing and maintenance}

Copepods were collected in June of 2016 from Esker Point Beach in Groton, CT, USA $\left(41.320725^{\circ} \mathrm{N}, 72.001643^{\circ} \mathrm{W}\right)$ and raised for at least three generations as stock cultures prior to the start of transgenerational experiments to limit maternal effects ${ }^{52}$. Stock cultures were split evenly into eight groups of 160 females and 80 males. Four of these eight groups were acclimatized to high temperature at 1 degree Celsius per day and used to seed the two high temperature treatments (OW and OWA). The other four groups remained at ambient temperature and were used to seed the ambient and acidification treatments. After temperature acclimatization, groups of stock cultures seeded the parental (F0) individuals for two days. Stock culture groups yielded an average of 7,173 eggs per group to produce approximately 57,000 parental (F0) eggs. Resulting parental eggs and N1 nauplii were acclimated to one of four experimental treatments over the entire F0 generation: 1) Ambient control (AM; temperature = $18^{\circ} \mathrm{C}, \mathrm{CO}_{2} \sim 400 \mu \mathrm{atm} ; \mathrm{pH} \sim 8.2$ ); 2) Ocean Acidification (OA; ambient temperature, high $\mathrm{CO}_{2}$ 2000 $\mu$ atm $\left.\mathrm{CO}_{2}, \mathrm{pH} \sim 7.5\right)$; 3) Ocean Warming (OW; high temperature $=22^{\circ} \mathrm{C}$, ambient $\mathrm{CO}_{2}$ );

4) combined warming and acidification (OWA; high temperature, high $\mathrm{CO}_{2}$ ). Treatment replicates were derived from the stock culture groups (i.e. stock culture group 1 after high temperature acclimatization seeded the F0 for OW replicate 1 and OWA replicate 1). The ambient temperature was chosen from a Gaussian fit-model ${ }^{53}$ for optimal recruitment (egg production $\times$ egg hatching success) vs temperature derived from Acartia tonsa populations from Casco Bay (Gulf of Maine), Long Island Sound, and Chesapeake Bay (USA), $R=$ $11.1 e^{-0.5\left(\frac{|T-17.7|}{6.4}\right)^{2}}$ where $\mathrm{R}$ is recruitment (egg production rate $\times$ hatching success), $\mathrm{T}$ is temperature $\left({ }^{\circ} \mathrm{C}\right), 17.7$ is the optimal temperature and 6.4 is the standard deviation around the 
313 optimal temperature ( $\mathrm{N}=54, \mathrm{r}^{2}=0.42$; our own unpublished data). Each treatment was kept in a

314 separate temperature-controlled incubator (Thermo FisherScientific ${ }^{\circledR}$ Isotemp ${ }^{\mathrm{TM}}$; Waltham, MA,

315 USA) and split into four replicate 10L culture containers (Cambro; Huntington Beach, CA,

316 USA). Copepods were fed every $48-72 \mathrm{~h}$ at food-replete concentrations $\left(\geq 800 \mu \mathrm{g}\right.$ Carbon $\left.\mathrm{L}^{-1}\right)$

317 consisting of equal proportions of the phytoplankters Tetraselmis sp., Rhodomonas sp., and

318 Thalassiosira weissflogii, following long-standing copepod culture protocols in our lab ${ }^{54}$. The

319 phytoplankton fed to copepods was deliberately raised under ambient conditions for the entire

320 length of the experiment to avoid confounding effects of possible changes in food quality due to

321 the different temperature and $\mathrm{CO}_{2}$ among treatments. We minimized the chance of selecting for

322 early developers in the cultures. Based on development time and adult longevity, we allowed

323 copepods to contribute progeny to the next generation for 7-10 days once we observed the first

324 nauplii of a new generation.

326 Elevated $\mathrm{CO}_{2}$ levels were achieved with gas proportioners (Cole-Parmer; Vernon Hills, IL, USA)

327 mixing laboratory air with $100 \%$ bone dry $\mathrm{CO}_{2}$ that was delivered continuously to the bottom of

328 each replicate culture. For small volume life-history trait experiments (sections b-d), $\mathrm{CO}_{2}$-mixed

329 air was fed into custom plexiglass enclosures within each temperature-controlled incubator to

330 allow for passive diffusion of $\mathrm{CO}_{2}$ into seawater. Target $\mathrm{pH}$ values were monitored using a

331 handheld $\mathrm{pH}$ probe (Orion Ross Ultra pH/ATC Triode with Orion Star A121 pH Portable Meter

332 (Thermo FisherScientific@; Waltham, MA, USA). The pH probe was calibrated monthly using

333 commercially available NBS pH standards in a three-point calibration ( $\mathrm{pH} 4.01,7,10.01$;

334 Thermo FisherScientific $\AA$; Waltham, MA, USA). Temperature and $\mathrm{pH}$ were monitored to ensure

335 that small-volume experiments in the plexiglass enclosures matched those of bulk cultures. To 
counteract metabolic $\mathrm{CO}_{2}$ accumulation, control $\mathrm{CO}_{2}$ conditions were achieved by forcing compressed ambient air through a series of $\mathrm{CO}_{2}$-stripping units containing granular soda lime (AirGas ${ }^{\circledR}$; Waterford, CT, USA) and a particle filter $(1 \mu \mathrm{m})$, and then to each culture container via airstone. Continuous bubbling maintained dissolved oxygen levels at $>8 \mathrm{mg} \mathrm{L}^{-1}$. Temperature, $\mathrm{pH}$, and actual $\mathrm{pCO}_{2}$ were monitored throughout the experiment (Supplementary Table 1). Actual $p \mathrm{CO}_{2}$ conditions were calculated in $\mathrm{CO}_{2} \mathrm{SYS}^{55}$ based on measurements of salinity, temperature, $\mathrm{pH}$, and total alkalinity $\left(A_{\mathrm{T}}\right.$; Supplementary Tables $\left.2-3\right)$ with $\mathrm{k} 1 / \mathrm{k} 2$ from Lueker, et al. $2000^{56}, \mathrm{KHSO}_{4}$ from Dickson $1990^{57}$, total Boron from Uppstrom $1974{ }^{58}$, and pH based on NBS scale. Total alkalinity was measured in triplicates three times over the course of the experiment using endpoint titration (G20 Potentiometric Titrator; Mettler Toledo $\left.{ }^{\circledR}\right)^{59}$.

Because treatments were housed in separate incubators, incubator-specific effects are theoretically possible, but unlikely ${ }^{60,61}$ given that incubators were held under identical ambient conditions except for the constant temperature and $\mathrm{CO}_{2}$ conditions that were meticulously monitored, and verified by independent measures throughout the experiment (Supplementary Table 5). Because of logistical and personnel constraints, egg production rate (EPR), hatching success (HS), survival, and development time (see below) could not be measured at every generation. Instead, measurements were taken at generations $0,3,6,9,12,15$, and 25 .

\section{b) Egg production rate and hatching success}

For each replicate culture within a treatment, 10 pairs of newly developed males and females were placed into $20 \mathrm{~mL}$ petri dishes for $48 \mathrm{~h}(\mathrm{n}=280$ per treatment). The dishes were housed in custom-made, airtight, plexiglass enclosures whose atmosphere was controlled to the appropriate 
$\mathrm{CO}_{2}$ concentration (see copepod culturing and maintenance section). There was one enclosure per temperature-controlled incubator. After the 48-h egg laying period, adults were checked for survival and removed from the petri dishes. Eggs were left in the dishes for an additional $72 \mathrm{~h}$ to allow for egg hatching and their contents preserved with non-acid Lugol's solution. Dishes with dead males were used for EPR, but not HS, since fertilization could not be assumed. Dishes with

363 dead females were discarded. EPR was calculated as $\frac{E_{u}+E_{h}}{t}$ where $E_{u}$ represents unhatched eggs,

$364 E_{h}$ represents hatched eggs (nauplii), and $t$ represents egg laying time. Hatching success was

365 calculated as $\frac{E_{h}}{E_{u}+E_{h}}$.

c) Survival generation, all adults from the previous generation were removed from the culture and allowed to lay eggs in food-replete media for $48 \mathrm{~h}$. Resulting nauplii were chosen for tracking survival. Unhatched eggs and any nauplii not chosen for survival analysis were returned to their respective replicate cultures for continued population maintenance. To measure survival for all generations

372 where life-history traits were evaluated, three $250-\mathrm{mL}$ beakers for each replicate culture were

373 supplied with 25 randomly chosen N1 nauplii each and housed in the plexiglass enclosure

374 described above $(n=21$ per treatment). Copepods were checked every $48-72 \mathrm{~h}$. The number of

375 dead, live, and missing copepods were logged for each beaker along with general stage (i.e.

376 nauplius, copepodite, adult female, or adult male). The fraction of survived individuals $\left(l_{x}\right)$ was

377 calculated as $n_{x} / n_{i}$ where $n_{x}$ represents the number of live individuals on day $x$, and $n_{i}$ represents

378 initial individuals. Nauplii were grown with media at levels of $500 \mu \mathrm{g} \mathrm{C} \mathrm{L}^{-1}$ to prevent

379 overgrowth of phytoplankton and allow for adequate nauplii grazing. Following the naupliar 
380 stages, copepods were grown with food-replete $\left(800 \mu \mathrm{g} \mathrm{C} \mathrm{L}^{-1}\right)$ media as described earlier. Food

381 was replaced with fresh media on monitoring days. Average survival was calculated per each

382 replicate culture at each generation measured. Differences in day-specific survival between

383 replicates and treatments was assessed using the 'survival' package in $\mathrm{R}^{62}$.

\section{d) Development time}

To calculate development time (time from N1 to adulthood) we recorded the number of days at which adults matured during the survival experiments and averaged the observations.

Sex ratio was calculated based on the number of surviving adult females relative to surviving adult males in survival experiments.

391 The population net reproductive rate, $\lambda$, was calculated as the dominant eigenvalue of an assembled projected age-structured Leslie Matrix constructed from survival and fecundity data ${ }^{37}$.

393 Briefly, day-specific probabilities of survival are calculated from day-specific survival as $P_{x}=$

$394 \frac{l_{x}}{l_{x-1}}$ where $l_{x}$ represents the proportion of individuals on day $x$ and $l_{x-1}$ represents the proportion 395 of individuals on day $x$-1. Probabilities of survival on day 1 are assumed to be $100 \%$ or a value 396 of "1.0". Per capita EPR and HS are calculated as described above, with fecundity rates equaling 397 the product of EPR and HS. Because only females produce offspring, total fecundity rates must 398 be scaled to the sex ratio (proportion of females:males). To account for differences in individual 399 development time for each treatment, fecundity rates are assigned to all days after the first matured adult is observed. We assume that surviving individuals represented by the survival 
401 experiments are equally as likely to experience any of the fecundity values observed in EPR

402 experiments. Therefore, each mate-pair fecundity rate was paired with each survival beaker to

403 construct a matrix. This yields a maximum of 120 matrices per treatment per generation (3

404 survival beakers $\times 4$ replicate cultures $\times 10$ mate pairs).

407 relative fitness $(\lambda / \bar{\lambda})$ against survival, egg production rate, hatching success, and sex ratio at both F0 and F25 for each treatment and calculating the partial regression coefficient estimate for each trait $^{63}$. The path analysis was completed by creating structural equation models $s^{43,44}$ of relative fitness against survival, egg production rate, hatching success, and sex ratio at both F0 and F25

411 for each treatment using the 'lavaan' and 'semPlot' packages in $\mathrm{R}^{64,65}$. Development time was 412 omitted from the models because the lack of variance within a replicate violated assumptions of 413 the model.

415 To quantify genetic diversity across the genome, capture probes were designed to target both 416 coding and regulatory regions across the genome. For coding regions, we chose the highest 417 quality probe falling within the region. Regulatory probes were set within 1,000 bp upstream of 418 the transcription start site. Genomic DNA was shipped to Rapid Genomics (Gainesvill, FL, 419 USA) for library preparation and was captured with the 32,413 probes $(21,311$ coding, 11,102 420 regulatory). Enriched libraries were sequenced on a HiSeq 4000 with 150 bp paired end reads.

421 Raw data were trimmed for quality and adapter contamination with Trimmomatic v0.36 ${ }^{66}$.

422 Trimmed reads were mapped to the $A$. tonsa reference genome ${ }^{67}$ with BWA-MEM ${ }^{68}$. 
423 SAMTOOLs ${ }^{69}$ was used to generate a pileup for each sample, from which genetic diversity $(\pi)$

424 was estimated with Popoolation ${ }^{70}$. We identified $1,450100 \mathrm{bp}$ windows in 704 unique scaffolds

425 across the genome that were present across all samples. We estimated $\pi$ in $100 \mathrm{bp}$ sliding

426 windows with a 100bp step size. Each position required a minimum coverage of $30 \times$, max

427 coverage of $1000 \times$ (to avoid mapping errors), and at least 0.5 of the window meeting these

428 thresholds. Resulting windows were required to be sequenced across all samples. To take into

429 account the independent replicates within each treatment, we used pairwise Wilcoxon Rank Sum

430 tests with a Holm correction for multiple testing. Genomic data is deposited in GenBank:

431 BioProject number PRJNA590963. All statistics were performed in $\mathrm{R}^{71}$.

433 Statistical analyses were computed using the software package R (v 4.0.2) ${ }^{71}$. To examine effects

434 of generation on changing life-history traits, we created trait-specific generalized additive models

435 (GAMs) smoothed across generations for each treatment ${ }^{72,73}$. To evaluate differences between

436 life-history traits, we created separate linear mixed models with replicates as random effects and

437 used post-hoc t-tests and Tukey HSD tests to compare life-history trait values that were

438 significantly different from other treatments at each generation $(\alpha<0.05)$. Analysis of fitness $(\lambda)$

439 calculations also included estimations with a zero-inflated generalized linear mixed effects

440 model with generation and treatment as fixed effects and replicates as random effects. Analyzing

441 the data with linear mixed models also allowed us to evaluate the effects of treatment replicates

442 on life-history traits. A low intra-class correlation coefficient suggests no predictive effect of

443 random variables. For the model constructed for fitness over generations, the variance due to

444 replicates within a treatment is very low $(\mathrm{ICC}=0.08$; Table 1$)$ and does not affect the model

445 results. To estimate the predicted probabilities of $\lambda=0$ across generations in Supplementary Fig. 
4464 , we converted $\lambda$ values to either 0 or 1 representing $\lambda$ values of 0 and $>0$, respectively. We used

447 the binomial-converted $\lambda$ data to fit a linear mixed effects model against generation and

448 treatment with replicates included as random effects. To evaluate individual effects of

449 temperature, $\mathrm{pH}$, or generation on life-history traits, we constructed a third linear model that was 450 tested with a three-way analysis of variance.

Acknowledgements: Research supported by grants from the USA National Science Foundation (OCE-1559180 awarded to HGD, MBF and HB and OCE-1559075 awarded to MHP) and

454 Connecticut Sea Grant ( R/LR-25) awarded to HGD, MBF, and HB.. The authors thank W.

455 Huffman for aiding in pilot experiments, C. Murray for assistance in alkalinity measurements, D.

456 Arbige, C. Woods, and B. Dziomba for help in maintaining equipment and constructing custom

457 enclosures for the experiments, and T. Moore and J. Lee of UConn's Statistical Consulting

458 Services for advice and assistance on data analysis.

Author Contributions: HGD conceived the project, designed research, aided in data analysis, and wrote the manuscript. JAD conducted experiments, analyzed data, created figures, and wrote

462 the manuscript with HGD. GP, LN, and XH conducted experiments. MBF conceived the project 463 and designed research. $\mathrm{HB}$ conceived the project, designed research, and designed the $\mathrm{CO}_{2}$

464 delivery system. RSB performed genomic diversity analysis. MHP conceived the project, 465 designed research, and performed genomic analysis. All authors edited and approved the paper.

467 Competing Interests: The authors declare no competing interests. 
469 Data and Script availability: The phenotypic data and R-scripts referred in the text have been

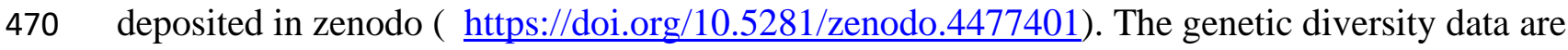

471 deposited in GenBank: BioProject number PRJNA590963, to be released upon publication of

472 this paper. 
474 1. Hönisch, B. et al. The Geological Record of Ocean Acidification. Science (80-. ). 335,

$475 \quad 1058-1063$ (2012).

476 2. Bindoff, N. L. et al. Changing Ocean, Marine Ecosystems, and Dependent Communities.

477 IPCC Spec. Rep. Ocean Cryosph. a Chang. Clim. 447-588 (2019).

478 3. Pörtner, H.-O. et al. IPCC, 2019. Technical Summary. IPCC Spec. Rep. Ocean Cryosph. a $479 \quad$ Chang. Clim. 35-74 (2019).

480 4. Caldeira, K. \& Wickett, M. E. Anthropogenic carbon and ocean pH. Nature 425, 365 (2003).

5. Cai, W. J. et al. Acidification of subsurface coastal waters enhanced by eutrophication. Nat. Geosci. 4, 766-770 (2011).

6. Wallace, R. B., Baumann, H., Grear, J. S., Aller, R. C. \& Gobler, C. J. Coastal ocean acidification: The other eutrophication problem. Estuar. Coast. Shelf Sci. 148, 1-13 (2014).

7. Radeloff, V. C. et al. The rise of novelty in ecosystems. Ecol. Appl. 25, 2051-2068 (2015).

8. Munday, P. L., Warner, R. R., Monro, K., Pandolfi, J. M. \& Marshall, D. J. Predicting evolutionary responses to climate change in the sea. Ecol. Lett. 16, 1488-1500 (2013).

9. Kelly, M. W. \& Hofmann, G. E. Adaptation and the physiology of ocean acidification. Funct. Ecol. 27, 980-990 (2013).

10. Pespeni, M. H. et al. Evolutionary change during experimental ocean acidification. Proc. Natl. Acad. Sci. U. S. A. 110, 6937-6942 (2013).

11. Thor, P. \& Dupont, S. Transgenerational effects alleviate severe fecundity loss during ocean acidification in a ubiquitous planktonic copepod. Glob. Chang. Biol. 21, 2261-2271 (2015).

12. Donelson, J. M., Salinas, S., Munday, P. L. \& Shama, L. N. S. Transgenerational plasticity and climate change experiments: Where do we go from here? Glob. Chang. Biol. 24, 1334 (2018).

13. Chevin, L. M., Lande, R. \& Mace, G. M. Adaptation, plasticity, and extinction in a changing environment: Towards a predictive theory. PLoS Biol. 8, (2010).

14. Angilletta, M. J. Thermal Adaptation: A Theoretical and Empirical Synthesis. (2009). doi:10.1093/acprof

15. Byrne, M. Impact of ocean warming and ocean acidification on marine invertebrate life history stages: Vulnerabilities and potential for persistence in a changing ocean. Oceanogr. Mar. Biol. An Annu. Rev. 49, 1-42 (2011).

16. Whiteley, N. M. Physiological and ecological responses of crustaceans to ocean acidification. Mar. Ecol. Prog. Ser. 430, 257-271 (2011). 
17. Cripps, G., Lindeque, P. \& Flynn, K. J. Have we been underestimating the effects of ocean acidification in zooplankton? Glob. Chang. Biol. 20, 3377-3385 (2014).

18. Baumann, H. Experimental assessments of marine species sensitivities to ocean acidification and co-stressors: How far have we come? Can. J. Zool. 97, 399-408 (2019).

19. Gibbin, E. M. et al. Can multi-generational exposure to ocean warming and acidification lead to the adaptation of life history and physiology in a marine metazoan? J. Exp. Biol.

20. Gibbin, E. M., Massamba N'Siala, G., Chakravarti, L. J., Jarrold, M. D. \& Calosi, P. The

21. Gonzalez, A., Ophelie, R., Ferriere, R. \& Hochberg, M. E. Evolutionary rescue: an emerging focus at the intersection between ecology and evolution. Philos. Trans. R. Soc. B Biol. Sci. 368, 1-8 (2012).

22. Bell, G. \& Gonzalez, A. Evolutionary rescue can prevent extinction following environmental change. Ecol. Lett. 12, 942-948 (2009).

23. Carlson, S. M., Cunningham, C. J. \& Westley, P. A. H. Evolutionary rescue in a changing world. Trends Ecol. Evol. 29, 521-530 (2014).

24. Hardy, A. The Open Sea: The World of Plankton. (Collins, 1970).

25. Huys, R. \& Boxshall, G. A. Copepod Evolution. (Ray Society, 1991).

26. Beaugrand, G. \& Reid, P. C. Long-term changes in phytoplankton, zooplankton and salmon related to climate. Glob. Chang. Biol. 9, 801-817 (2003).

27. Möllmann, C., Müller-Karulis, B., Kornilovs, G. \& St John, M. A. Effects of climate and overfishing on zooplankton dynamics and ecosystem structure: Regime shifts, trophic cascade, and feedback loops in a simple ecosystem. ICES J. Mar. Sci. 65, 302-310 (2008).

28. Steinberg, D. K. \& Landry, M. R. Zooplankton and the Ocean Carbon Cycle. Annu. Rev. Mar. Sci. 9, 413-444 (2017).

29. Mauchline, J. The Biology of Calanoid Copepods. in Advances in Marine Biology 33 (1998).

30. Turner, J. T. The feeding ecology of some zooplankters that are important prey items of larval fish. (1984).

31. Rice, E., Dam, H. G. \& Stewart, G. Impact of Climate Change on Estuarine Zooplankton: Surface Water Warming in Long Island Sound Is Associated with Changes in Copepod Size and Community Structure. Estuaries and Coasts 1-11 (2014). doi:10.1007/s12237014-9770-0

32. Gobler, C. J. \& Baumann, H. Hypoxia and acidification in marine ecosystems : Coupled dynamics and effects on ocean life Hypoxia and acidification in ocean ecosystems : coupled dynamics and effects on marine life. Biol. Lett. 12, (2016).

33. Côté, I. M., Darling, E. S. \& Brown, C. J. Interactions among ecosystem stressors and 
their importance in conservation. Proc. R. Soc. B Biol. Sci. 283, 1-9 (2016).

34. Burt, A. Perspective: The Evolution of Fitness. Evolution (N. Y). 49, 1-8 (1995).

35. Hendry, A. P. \& Gonzalez, A. Whither adaptation? Biol. Philos. 23, 673-699 (2008).

36. Arnold, S. J., Pfrender, M. E. \& Jones, A. G. The adaptive landscape as a conceptual bridge between micro- and macroevolution. Genetica 112-113, 9-32 (2001).

37. Caswell, H. Matrix Population Models: Construction, Analysis, and Interpretation. (Sinauer Associates, Inc., 2001).

38. Sasaki, M. C. \& Dam, H. G. Integrating patterns of thermal tolerance and phenotypic plasticity with population genetics to improve understanding of vulnerability to warming in a widespread copepod. Glob. Chang. Biol. 25, 4147-4164 (2019).

39. Luikart, G., England, P. R., Tallmon, D., Jordan, S. \& Taberlet, P. The power and promise of population genomics: From genotyping to genome typing. Nat. Rev. Genet. 4, 981-994 (2003).

40. Black IV, W. C., Baer, C. F., Antolin, M. F. \& DuTeau, N. M. Population genomics: Genome-wide sampling of insect populations. Annu. Rev. Entomol. 46, 441-469 (2001).

41. Brennan, R. et al. Loss and recovery of transcriptional plasticity after long-term adaptation to global change conditions in a marine copepod. bioRxiv (2021). doi: https://doi.org/10.1101/2020.01.29.925396

42. Kingsolver, J. G. \& Pfennig, D. W. Patterns and Power of Phenotypic Selection in Nature. Bioscience 57, 561-572 (2007).

43. Crespi, B. J. \& Bookstein, F. L. A Path-Analytic Model for the Measurement of Selection on Morphology. Evolution (N. Y). 43, 18 (1989).

44. Pigliucci, M. \& Kaplan, J. Making Sense of Evolution. (2013). doi:10.7208/chicago/9780226668352.001.0001

45. Bush, A. et al. Incorporating evolutionary adaptation in species distribution modelling reduces projected vulnerability to climate change. Ecol. Lett. 19, 1468-1478 (2016).

46. Riebesell, U. \& Gattuso, J. Lessons learned from ocean acidification research. Nat. Clim. Chang. 5, 2014-2016 (2015).

47. De Wit, P., Dupont, S. \& Thor, P. Selection on oxidative phosphorylation and ribosomal structure as a multigenerational response to ocean acidification in the common copepod Pseudocalanus acuspes. Evol. Appl. 9, 1112-1123 (2016).

48. Chakravarti, L. J. et al. Can trans-generational experiments be used to enhance species resilience to ocean warming and acidification? Evol. Appl. 9, 1133-1146 (2016).

49. Carrier-Belleau, C., Drolet, D., Mckindsey, C. W. \& Archambault, P. Environmental stressors , complex interactions and marine benthic communities ' responses. Sci. Rep. 114 (2021). doi:10.1038/s41598-021-83533-1

50. Dam, H. G. \& Baumann, H. Climate Change, Zooplankton and Fisheries. Clim. Chang. 
Impacts Fish. Aquac. 851-874 (2017). doi:10.1002/9781119154051.ch25

585

586

587

588

589

590

591

592

593

594

595

596

597

598

599

600

601

602

603

604

605

606

607

608

609

610

611

612

613

614

615

616

617

618

619

620

51. Bell, G. Evolutionary rescue and the limits of adaptation. Philos. Trans. R. Soc. B Biol. Sci. 368, 1-6 (2013).

52. Falconer, D. S. Introduction to Quantitative Genetics. (Longman Scientific and Technical, 1989).

53. Angilletta Jr, M. J. Estimating and comparing thermal performance curves. J. Therm. Biol. 31, 541-545 (2006).

54. Feinberg, L. R. \& Dam, H. G. Effects of diet on dimensions, density and sinking rates of fecal pellets of the copepod Acartia tonsa. Mar. Ecol. Prog. Ser. 175, 87-96 (1998).

55. Pierrot, D., Lewis, E. \& Wallace, D. W. R. MS Excel Program Developed for CO2 System Calculations. (2006). doi:10.3334/CDIAC/otg.CO2SYS_XLS_CDIAC105a

56. Lueker, T. J., Dickson, A. G. \& Keeling, C. D. Ocean pCO2 calculated from dissolved inorganic carbon, alkalinity, and equations for K1 and K2: validation based on laboratory measurements of CO2 in gas and seawater at equilibrium. Mar. Chem. 70, 105-119 (2000).

57. Dickson, A. G. Standard potential of the reaction: $\mathrm{AgCl}(\mathrm{s})+12 \mathrm{H} 2(\mathrm{~g})=\mathrm{Ag}(\mathrm{s})+\mathrm{HCl}(\mathrm{aq})$, and the standard acidity constant of the ion HSO4- in synthetic sea water from 273.15 to 318.15K. J. Chem. Thermodyn. 22, 113-127 (1990).

58. Uppstrom, L. R. The boron/chlorinity ratio of deep-sea water from the Pacific Ocean. Deep. Res. Oceanogr. Abstr. 21, 161-162 (1974).

59. Murray, C. S. \& Baumann, H. You better repeat it: Complex CO2x temperature effects in Atlantic silverside offspring revealed by serial experimentation. Diversity 10, (2018).

60. Schank, J. C. \& Koehnle, T. J. Pseudoreplication is a Pseudoproblem. J. Comp. Psychol. 123, 421-433 (2009).

61. Oksanen, L. Logic of experiments in ecology: is pseudoreplication a pseudoissue ? Oikos 94, 27-38 (2001).

62. Therneau, T._A Package for Survival Analysis in $S_{-}$. (2015).

63. Lande, R. \& Arnold, S. J. The Measurement of Selection on Correlated Characters. Evolution (N. Y). 37, 1210 (1983).

64. Rosseel, Y. Lavaan: An R package for structural equation modeling. J. Stat. Softw. 48, (2012).

65. Epskamp, S., Stuber, S., Nak, J., Veenman, M. \& Jorgensen, T. D. semPlot: Path Diagrams and Visual Analysis of Various SEM Packages' Output. (2019).

66. Bolger, A. M., Lohse, M. \& Usadel, B. Trimmomatic: A flexible trimmer for Illumina sequence data. Bioinformatics 30, 2114-2120 (2014).

67. Jørgensen, T. S. et al. The genome and mRNA transcriptome of the cosmopolitan calanoid copepod acartia tonsa dana improve the understanding of copepod genome size evolution. 
Genome Biol. Evol. 11, 1440-1450 (2019).

622 68. Li, H. Aligning sequence reads, clone sequences and assembly contigs with BWA-MEM.

623 00, 1-3 (2013).

624 69. Li, H. et al. The Sequence Alignment/Map format and SAMtools. Bioinformatics 25,

625 2078-2079 (2009).

626 70. Kofler, R. et al. Popoolation: A toolbox for population genetic analysis of next generation 627 sequencing data from pooled individuals. PLoS One 6, (2011).

628 71. R Core Team. R: A language and environment for statistical computing. (2013).

629 72. Wood, S. N. Fast stable restricted maximum likelihood and marginal likelihood estimation

630 of semiparametric generalized linear models. J. R. Stat. Soc. Ser. B Stat. Methodol. 73, 3-

631 36 (2011).

632 73. Simpson, G. L. Modelling palaeoecological time series using generalised additive models. $633 \quad$ Front. Ecol. Evol. 6, 1-21 (2018).

634

635 
Figure Legends:

Figure 1 - Mean egg production and mean hatching success for transgenerational

experiment. Egg production rate (EPR, histograms, left Y axis) and egg hatching success (HS,

(top left) - ambient temperature $\left(18^{\circ} \mathrm{C}\right)$ and $\left.\mathrm{CO}_{2}(400 \mu \mathrm{atm}) ; \mathbf{B}\right)$ Ocean acidification conditions,

OA: Green (top right) - ambient temperature and high $\mathrm{CO}_{2}(2000 \mu \mathrm{atm})$; C) Ocean warming

642

conditions, OW: Orange (bottom left) - high temperature $\left(22^{\circ} \mathrm{C}\right)$ and ambient $\left.\mathrm{CO}_{2} ; \mathbf{D}\right)$ Ocean

643

warming and acidification, OWA: Red (bottom right) - high temperature and high $\mathrm{CO}_{2}$. Error

644

bars represent $95 \%$ confidence intervals. Probability (p) values for EPR and HS for each panel

645

are derived from the effect of generation on each trait taken from generalized additive models.

646

Letters represent statistically similar groupings for post-hoc t-test comparison. Box and whiskers

647 plots of the same graphs are displayed on SI Fig. 6.

Figure 2 - Survival for transgenerational study. Mean survival is for nauplius stage 1 to adult 0.001). Treatment colors are the same as histograms in Fig. 1. Treatment curves are offset for

Figure 3 - Fitness. Mean fitness values, $\lambda$, calculated for the transgenerational study. Error bars represent $95 \%$ confidence intervals. Treatment colors are the same as histograms in Fig. 1.

656 Treatment curves are offset for clarity. Box and whiskers plots of the same graphs are displayed on SI Fig. 8. 
658 Figure 4 - Fitness landscapes. Frequency distributions (left axes) and relative fitness landscape

659 linear models (right axes) for A) Hatching success, B) Egg production rate, and C) Survival at F0

660 (black) and F25 (red). Fitness landscapes estimate the correlations between changing quantitative

661 phenotypes and changing relative fitness. Shading around lines represents standard error.

662

663 
664 Tables:

665

666

Table 1 - Generational effects on fitness. Zero-inflated model results of generation and treatment

667 on fitness, with replicates as random effects. Count model results represents when $\lambda=0$ values are

668 omitted. Control treatment is the reference intercept for the model. Interactive effect of generation

669 on AM conditions is noted as just "Generation" for each model results.

670

\begin{tabular}{|c|c|c|c|}
\hline Predictors & Estimates & $C I$ & $P$ \\
\hline \multicolumn{4}{|l|}{ Count model } \\
\hline (Intercept) & 1.19 & $1.16-1.23$ & $<0.001$ \\
\hline Generation & -0.00 & $-0.00--0.00$ & 0.001 \\
\hline$O A$ & 0.01 & $-0.04-0.06$ & 0.817 \\
\hline$O W$ & 0.04 & $-0.01-0.09$ & 0.115 \\
\hline$O W A$ & 0.13 & $0.07-0.18$ & $<0.001$ \\
\hline Generation $\times O A$ & 0.00 & $-0.00-0.00$ & 0.951 \\
\hline Generation $\times O W$ & 0.00 & $0.00-0.00$ & 0.009 \\
\hline Generation $\times O W A$ & -0.01 & $-0.01--0.01$ & $<0.001$ \\
\hline \multicolumn{4}{|l|}{ Zero-inflated model } \\
\hline (Intercept) & -1.97 & $-2.62--1.32$ & $<0.001$ \\
\hline Generation & -0.01 & $-0.04-0.03$ & 0.681 \\
\hline$O A$ & 0.55 & $-0.36-1.46$ & 0.233 \\
\hline$O W$ & 1.27 & $0.39-2.16$ & 0.005 \\
\hline$O W A$ & 1.54 & $0.65-2.44$ & 0.001 \\
\hline Generation $\times O A$ & -0.03 & $-0.07-0.02$ & 0.212 \\
\hline Generation $\times O W$ & -0.04 & $-0.09--0.00$ & 0.038 \\
\hline Generation $\times O W A$ & -0.19 & $-0.25--0.13$ & $<0.001$ \\
\hline \multicolumn{4}{|l|}{ Random effects } \\
\hline$\sigma^{2}$ & 0.01 & & \\
\hline$\tau_{00 \text { Replicate }}$ & 0.00 & & \\
\hline Intra-correlation coefficient & 0.08 & & \\
\hline N Replicate & 16 & & \\
\hline Observations & 2867 & & \\
\hline Marginal $R^{2} /$ Conditional $R^{2}$ & $0.230 / 0.293$ & & \\
\hline
\end{tabular}

671

672 
673 Table 2 - Path analysis and effects on lambda. Standardized parameter estimates from structural

674 equation models for each trait's effect on lambda at both F0 (gray bars) and F25 (white bars) for

675 each treatment. The highest parameter estimate for each treatment at each generation is highlighted

676 in bold.

677

678

679

680

\begin{tabular}{|c|c|c|c|c|}
\hline & $\mathbf{A M}$ & OA & OW & OWA \\
\hline \multirow{2}{*}{ Survival } & 0.12 & -0.09 & 0.03 & 0.08 \\
\hline & -0.09 & 0.13 & 0.72 & 0.33 \\
\hline \multirow{2}{*}{$E P R$} & -0.07 & 0.04 & 0.03 & 0.10 \\
\hline & 0.34 & 0.25 & 0.86 & 0.23 \\
\hline \multirow{2}{*}{$H S$} & 0.91 & 0.94 & 0.96 & 0.78 \\
\hline & 0.53 & 0.65 & 0.11 & 0.70 \\
\hline \multirow{2}{*}{$\begin{array}{c}\text { Sex } \\
\text { ratio }\end{array}$} & 0.12 & -0.15 & 0.09 & 0.21 \\
\hline & -0.39 & 0.37 & 0.37 & -0.27 \\
\hline
\end{tabular}




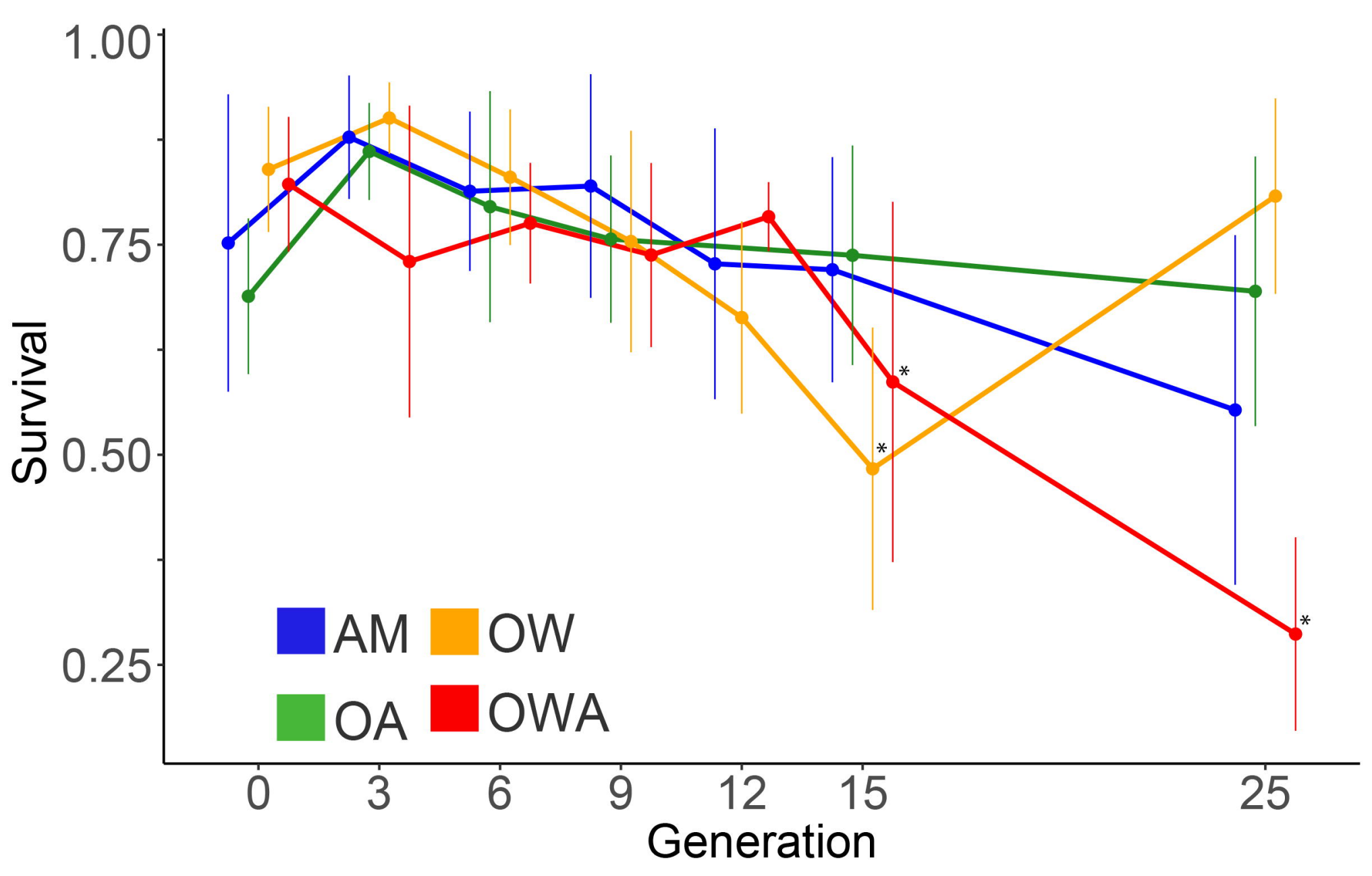


